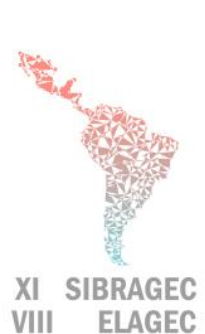

VIII ELAGEC
XI SIMPÓSIO BRASILEIRO DE GESTÃO E ECONOMIA DA CONSTRUÇÃO

VIII ENCUENTRO LATINOAMERICANO DE GESTIÓN

Y ECONOMÍA DE LA CONSTRUCCIÓN

Do conhecimento à ação: práticas avançadas de gestão da produção

Londrina, Paraná, Brasil. 23 a 25 de Outubro de 2019

\title{
INDICADORES DA QUALIDADE PARA SUSTENTABILIDADE DE OBRAS DE EDIFICAÇÕES: BANCO DE DADOS E VALORES DE REFERÊNCIA
}

\author{
FERNANDES, Luara L. A. (1); COSTA, Dayana B. (2); GUEDES, Henrique N. \\ (3); SIMÕES, Caio K. M. (4) \\ (1) Universidade Federal da Bahia, e-mail: luara.fernandes@gmail.com (2) Universidade Federal da \\ Bahia, e-mail: dayanabcosta@ufba.br (3) Universidade Federal da Bahia, e-mail: \\ henriqueguedes1029@gmail.com (4) Universidade Federal da Bahia, e-mail: caiosimoes23@gmail.com
}

\begin{abstract}
The construction sector has an important role in the global economy, but it is one of those that cause more environmental impacts. The sector has been concerned to sustainability issues and has used performance measurement and sustainability indicators to assist the environmental performance improvement. In Brazil, since 2012, the SIAC regulation of the Brazilian Program for Productivity and Quality of the Built Environment - PBQP-H started to require the collection of six sustainability indicators: waste generation throughout and at the end of the construction; water consumption throughout and at the end of the construction; energy consumption throughout and at the end of the construction. However, only the collection of the indicators do not generate performance improvement and reference values to guide the companies' goals. Furthermore, this paper refers to an on-going research which aims to establish a data base and benchmark values for the six PBQP-H sustainability indicators aiming to provide information for construction managers make decisions concerning the environmental quality performance of construction sites. The study was developed from a data survey conducted in seven Brazilian states. The main contributions of this study are the reference values presented for the indicators throughout the construction in each $10 \%$ of construction progress and the reference values presented for the indicators at the end of the construction.
\end{abstract}

Keywords: Sustainability indicators, Construction sites, PBQP-H, Performance measurement.

\section{INTRODUÇÃ̃O}

A construção é uma grande indústria na economia global (YUN; JUNG, 2017). Entretanto, pouca atenção vem sendo dada para as técnicas de construção e conservação ambiental (VASQUEZ et al., 2011). Assim, há uma necessidade contínua de entender soluções inovadoras na escala da construção as quais podem contribuir para uma cidade ambientalmente amigável e que conserve os recursos (YIN et al., 2018).

Medições de sustentabilidade no setor da construção estão ganhando muita atenção ao redor do mundo (BERARDI, 2012). No Brasil, o Programa Brasileiro de Qualidade e Produtividade do Habitat (PBQP-H) tem como objetivo apoiar o esforço brasileiro de modernidade e promover a qualidade e produtividade do setor da construção habitacional (MINISTÉRIO DAS CIDADES, 2018). Dentro do PBQP-H, o regimento

FERNANDES, L.L.A.; COSTA, D.B.; GUEDES, H.N.; SIMÕES, C.K.M. Indicadores da qualidade para sustentabilidade de obras de edificações: banco de dados e valores de referência. In: SIMPÓSIO BRASILEIRO DE GESTÃO E ECONOMIA DA CONSTRUÇÃO, 11., 2019,

Londrina. Anais [...]. Porto Alegre: ANTAC, 2019. Disponível em:

https://www.antaceventos.net.br/index.php/sibragec/sibragec2019/paper/view/439 
do SIAC (Sistema de Avaliação da Conformidade de Empresas de Serviços e Obras da Construção Civil) em 2012 passou a solicitar, das empresas certificadas, a coleta de seis indicadores da qualidade voltados à sustentabilidade durante a etapa de construção e no final da obra, sendo eles: indicador de geração de resíduos ao longo da obra; indicador de geração de resíduos ao final da obra; indicador de consumo de água ao longo da obra; indicador de consumo de água ao final da obra; indicador de consumo de energia ao longo da obra; indicador de consumo de energia ao final da obra. Na atualização de 2018 do regimento do SIAC, os indicadores ao longo da obra foram retirados dessa lista, sendo apenas solicitada a coleta dos indicadores ao final da obra.

Entretanto, os indicadores sozinhos não conseguem promover melhoria, sendo necessárias metas e referências de desempenho para cada indicador, para determinar a escala de desempenho e avaliar o progresso (SILVA, 2003). Nesse contexto, esse estudo tem como objetivo construir uma base de dados nacional e estabelecer valores de valores de referência para os seis indicadores de sustentabilidade do PBQP-H, visando oferecer suporte ao setor da construção no processo de tomada de decisão quanto a melhoria do seu desempenho em termos de qualidade ambiental.

Este trabalho está em desenvolvimento e faz parte de um projeto mais amplo patrocinado pela Câmera Brasileira da Indústria da Construção (CBIC), com apoio da Universidade Federal do Ceará, Universidade Federal de Goiás, Universidade Federal do Rio Grande do Sul e executado pela Universidade Federal da Bahia.

\section{METODOLOGIA}

A abordagem metodológica desta pesquisa é o levantamento de dados, uma vez que estão sendo levantados, em território nacional, os valores dos indicadores de sustentabilidade do PBQP-H, visando aumentar a compressão do desempenho da qualidade ambiental das obras de edificações.

A pesquisa teve uma etapa piloto com uma amostra de 20 obras da Bahia (COSTA et al, 2018). Na etapa atual, estão sendo coletados valores dos indicadores e de boas práticas relacionadas ao consumo de água, consumo de energia e geração de resíduos nos estados Bahia, Sergipe, Alagoas, Pernambuco, Ceará e Goiás e no Distrito Federal, conforme as três etapas a seguir: Seleção da amostra (1), Coleta de dados (2) e Análise dos dados (3) (Figura 1). 
SIBRAGEC - ELAGEC 2019 - de 23 a 25 de Outubro - LONDRINA - PR

Figura 1 - Delineamento do estudo

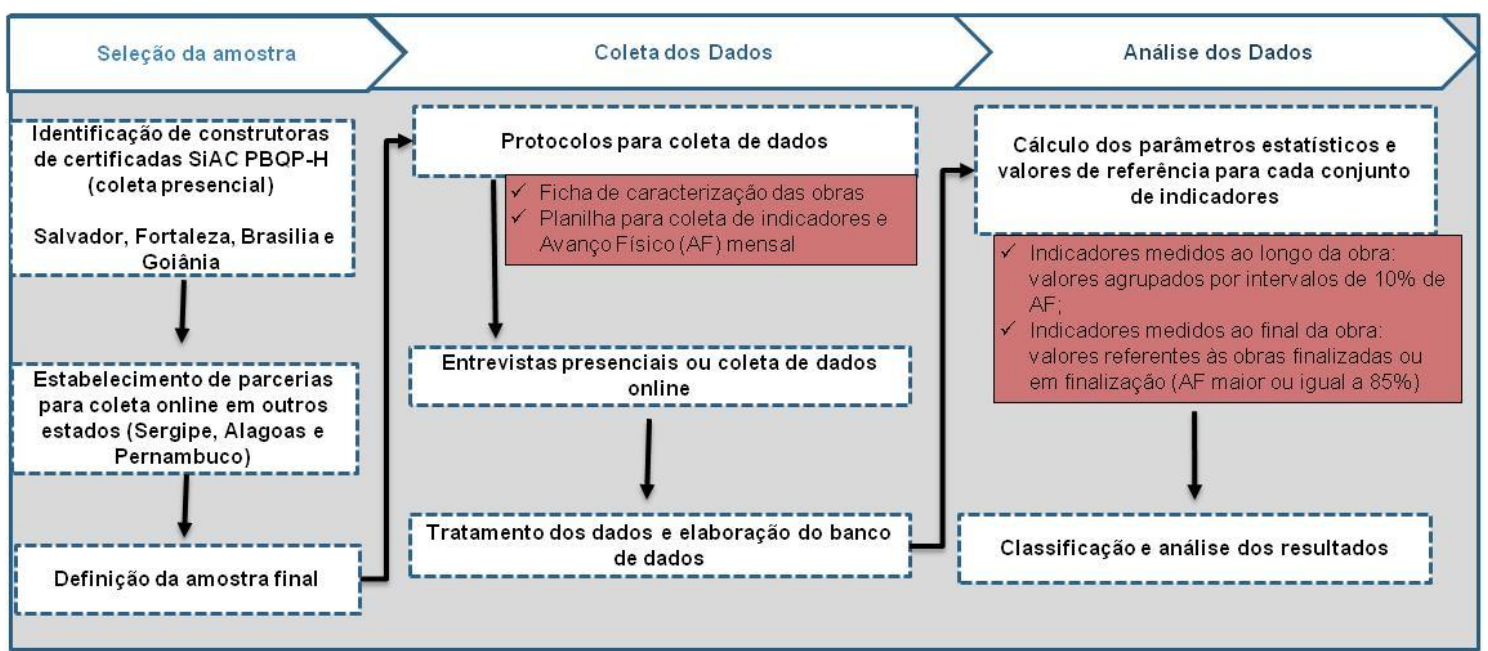

Fonte: Autores

A primeira etapa do estudo consistiu em identificar as empresas certificadas pelo PBQP$\mathrm{H}$ nas cidades selecionadas para a coleta. Estas cidades foram selecionadas devido à parcerias institucionais com as universidades locais e que desenvolvem estudos na área de gestão das construções, facilitando o contato com as empresas. A CBIC e o PBQP-H também forneceram uma lista de empresas certificadas nas cidades selecionadas, visando identificar a população adequada para a pesquisa. Após estabelecimento de contato com essas empresas, foi possível também alinhar parcerias para coletar dados de forma online em Sergipe, Alagoas e Pernambuco, a partir de empresa parceira da UFBA, sendo definida a amostra para o estudo.

A segunda etapa consistiu na coleta de dados e foi iniciada com a revisão dos protocolos de coleta do estudo piloto, seguida da coleta de dados online e presencial, por meio de missões de trabalho em Fortaleza, Brasília e Goiânia. Para o projeto, houve mais duas missões em Porto Alegre e em Campinas, que não entraram no escopo desse estudo. A etapa de processamento e análise dos dados está em desenvolvimento e, até o momento da redação deste artigo, foram realizadas apenas análises preliminares de 87 obras cadastradas no banco de dados.

Para as análises dos indicadores ao longo da obra, foram utilizados dados de 40 obras, sendo $60 \%$ delas de parede de concreto, $15 \%$ de alvenaria estrutural e $22 \%$ de concreto armado moldado in loco e 3\% de estrutura pré-moldada. Nessa amostra, 50\% das obras são da Bahia, $17 \%$ do Ceará, $12 \%$ de Alagoas, $8 \%$ de Sergipe e 13\% de Pernambuco. A Figura 2 apresenta a caracterização dessa amostra. 
SIBRAGEC - ELAGEC 2019 - de 23 a 25 de Outubro - LONDRINA - PR

Figura 2 - Caracterização da amostra dos indicadores ao longo da obra
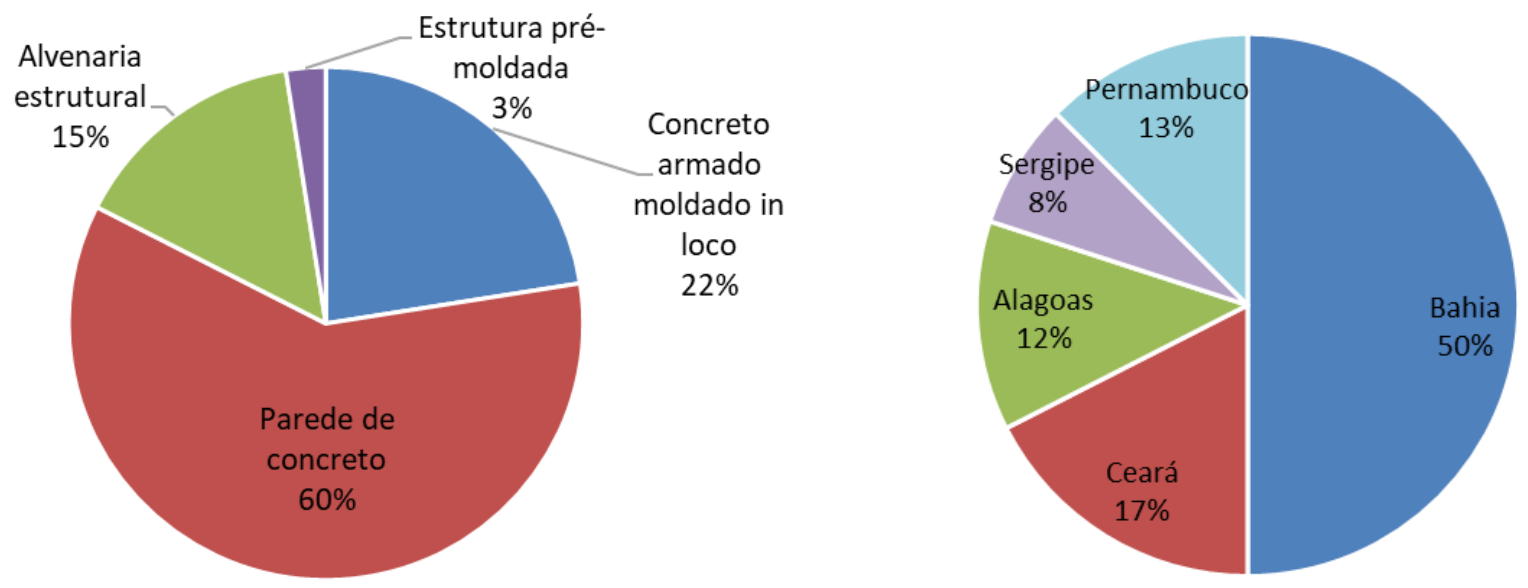

Fonte: Autores

Para os indicadores ao final de obra, foram consideradas obras completamente finalizadas e com avanço físico a partir de $85 \%$. Foi utilizada uma amostra de 31 obras, nas quais $32 \%$ das obras são de parede de concreto, $13 \%$ de alvenaria estrutural, $52 \%$ de concreto armado moldado in loco e $3 \%$ de estrutura pré-moldada. Nessa amostra, 58\% das obras são da Bahia, 3\% de Sergipe, 3\% de Alagoas, 13\% do Ceará, 3\% do Distrito Federal, $7 \%$ de Pernambuco e $13 \%$ de Goiás. A Figura 3 apresenta a caracterização dessa amostra.

\section{Figura 3 - Caracterização da amostra dos indicadores ao final da obra}
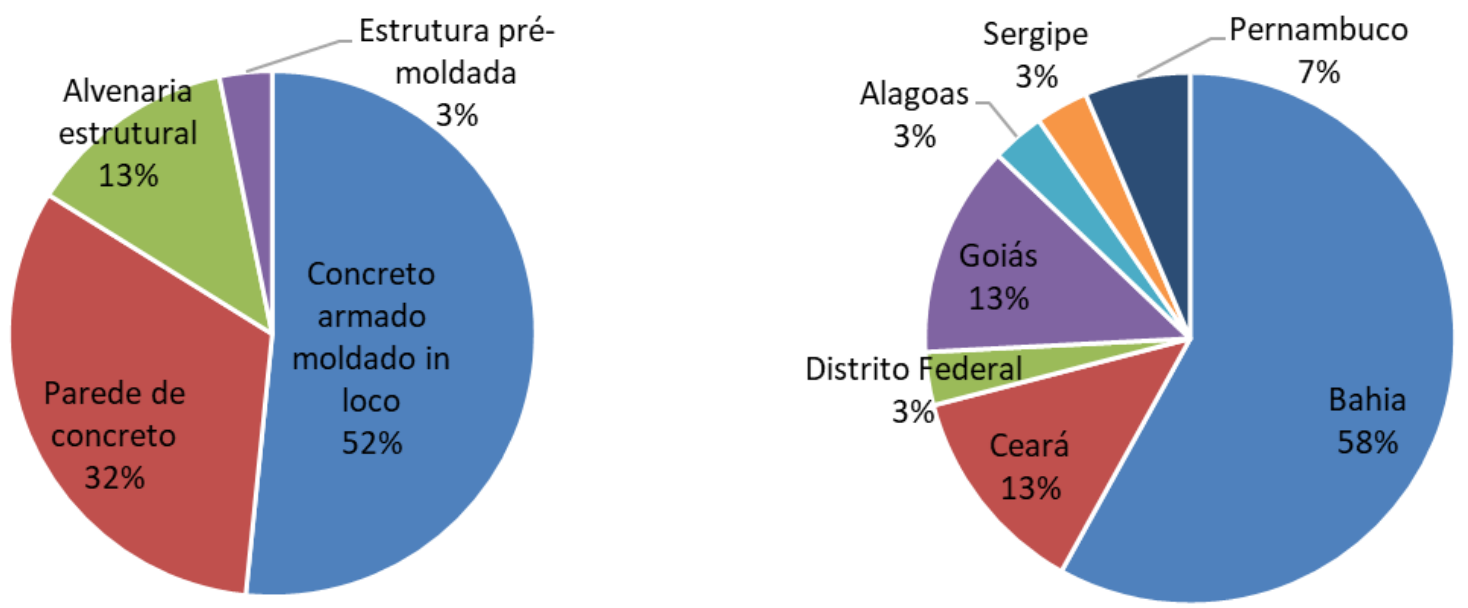

Fonte: Autores

\section{RESULTADOS}

Nesta seção serão apresentados os resultados preliminares obtidos para valores de referência para os seis indicadores de sustentabilidade do PBQP-H. Os indicadores ao longo da obra têm seus resultados apresentados por faixas de $10 \%$ de avanço físico e, para os indicadores ao final da obra, foram apresentados valores de referência gerais. 
SIBRAGEC - ELAGEC 2019 - de 23 a 25 de Outubro - LONDRINA - PR

\subsection{Consumo de água ao longo da obra (m³/trabalhador)}

A Figura 4 apresenta os valores de referência para o indicador de consumo de água ao longo da obra, calculados para faixas de avanço físico de $10 \%$.

Figura 4 - Valores de referência do indicador de consumo de água ao longo da obra para faixas de avanço físico de $10 \%$

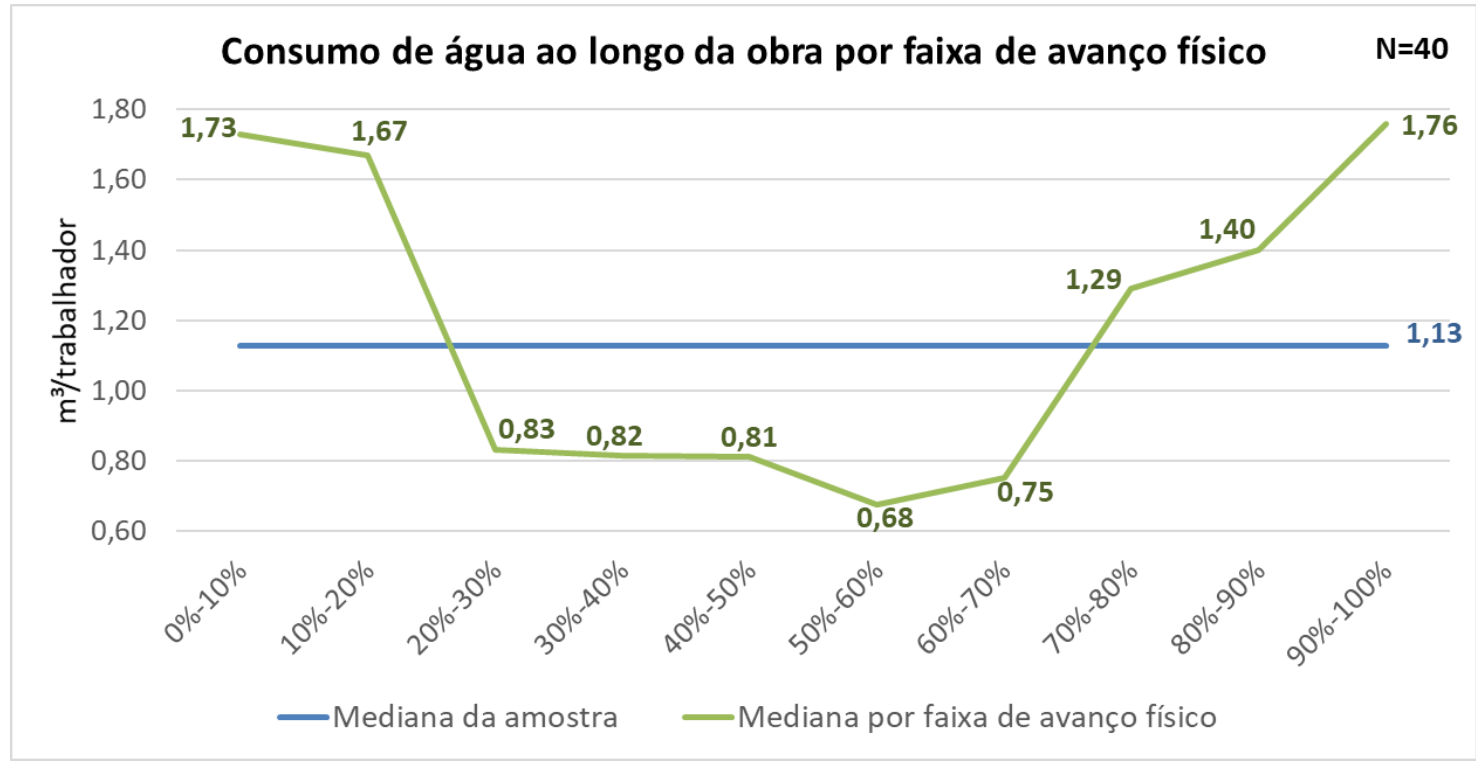

Fonte: Autores

Em relação ao consumo de água, observou-se uma variação das medianas entre 0,68 $\mathrm{m}^{3} /$ trabalhador e $1,76 \mathrm{~m}^{3} /$ trabalhador. Verifica-se que os maiores consumos estão associados às faixas de $0-10 \%$ e $90-100 \%$ de avanço físico. Esses resultados podem ser justificados pela baixa quantidade de trabalhadores em canteiros no início e no final da obra (uma vez que o indicador é inversamente proporcional ao efetivo médio), bem como a ocorrência de serviços de terraplanagem, limpeza final de obra e testes de estanqueidade.

O menor valor de mediana se apresentou na faixa de avanço entre 50-60\%, que em geral está associado à fase de estrutura nas obras. Esses resultados podem ser justificados pelo fato das estruturas da maioria das obras da amostra serem predominantemente de concreto comprados já usinados, isto é, o gasto de água na produção não é contabilizado na obra.

\subsection{Consumo de energia ao longo da obra ( $\mathrm{kWh} /$ trabalhador $)$}

A Figura 5 apresenta os valores de referência para o indicador de consumo de energia ao longo da obra, calculados para faixas de avanço físico de $10 \%$. 
SIBRAGEC - ELAGEC 2019 - de 23 a 25 de Outubro - LONDRINA - PR

Figura 5 - Valores de referência do indicador de consumo de água ao longo da obra para faixas de avanço físico de $\mathbf{1 0 \%}$

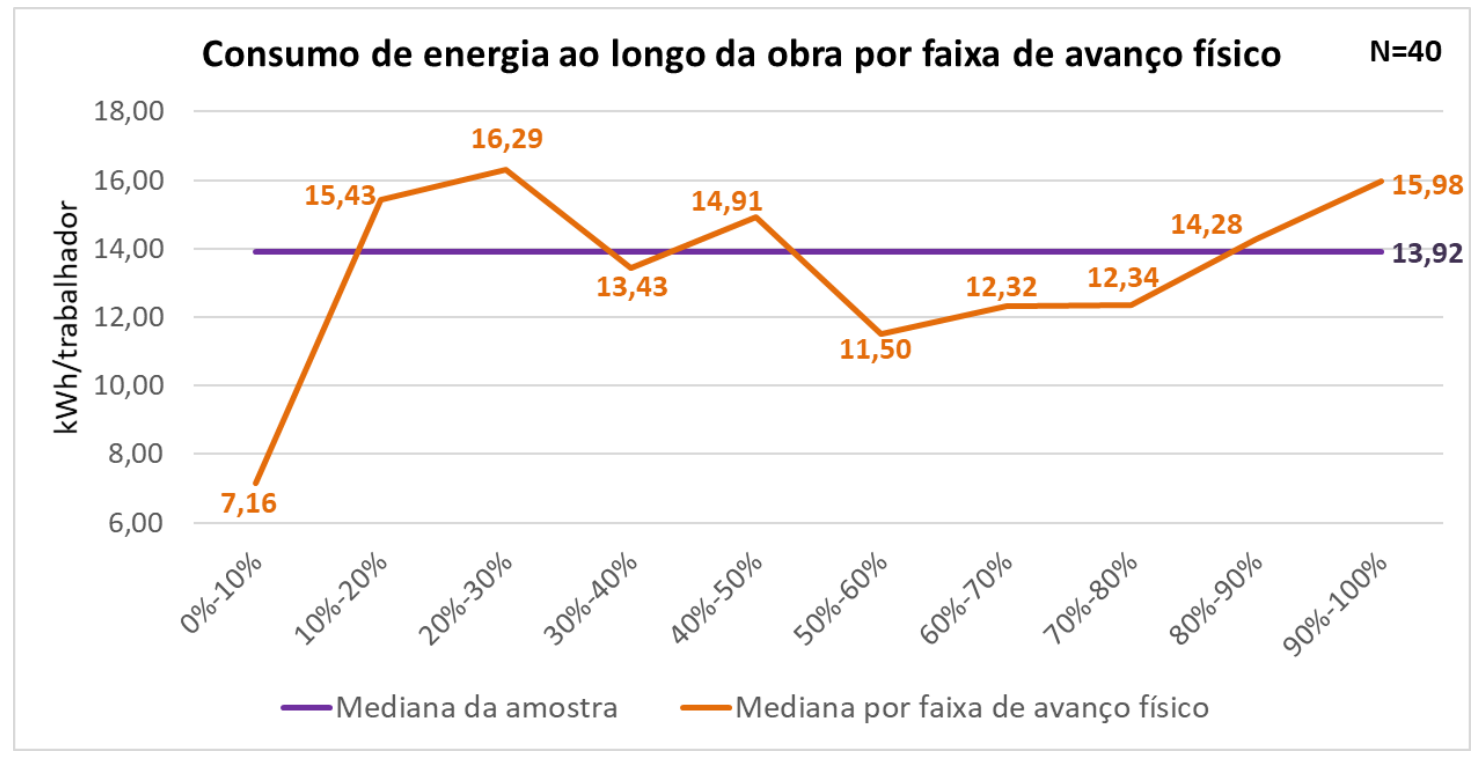

Fonte: Autores

Em relação ao consumo de energia ao longo da obra, observou-se uma variação das medianas entre $7,16 \mathrm{kWh} /$ trabalhador e $16,29 \mathrm{~m}^{3} /$ trabalhador. Verifica-se que o menor valor entre as medianas está associado à faixa de avanço físico de $0-10 \%$, o que se mostra coerente, devido ao fato de serem as fases iniciais da obra onde não se verifica uso massivo de equipamentos. A partir da faixa de avanço de 10-20\%, observa-se valores de medianas muito próximos, variando de 11,50 kWh/trabalhador à 16,29 $\mathrm{kWh}$ /trabalhador, mostrando uma tendência de não haver grandes variações no consumo de energia a partir dessa faixa.

O valor de mediana mais alto é $16,29 \mathrm{kWh} /$ trabalhador, na faixa de $20-30 \%$, que pode ser justificado pela instalação de elevadores de carga em obras verticais, demandando um maior consumo de energia no início do uso. O segundo maior valor de mediana encontrado foi $15,98 \mathrm{kWh} /$ trabalhador, referente à faixa de avanço de $90-100 \%$, podendo ser associado à fase de entrega da obra e teste de equipamentos.

\subsection{Geração de resíduos ao longo da obra ( $\mathrm{m}^{3} /$ trabalhador)}

A Figura 6 apresenta os valores de referência para o indicador de geração de resíduos ao longo da obra, calculados para faixas de avanço físico de $10 \%$. 
SIBRAGEC - ELAGEC 2019 - de 23 a 25 de Outubro - LONDRINA - PR

Figura 6 - Valores de referência do indicador de geração de resíduos ao longo da obra para faixas de avanço físico de $\mathbf{1 0 \%}$

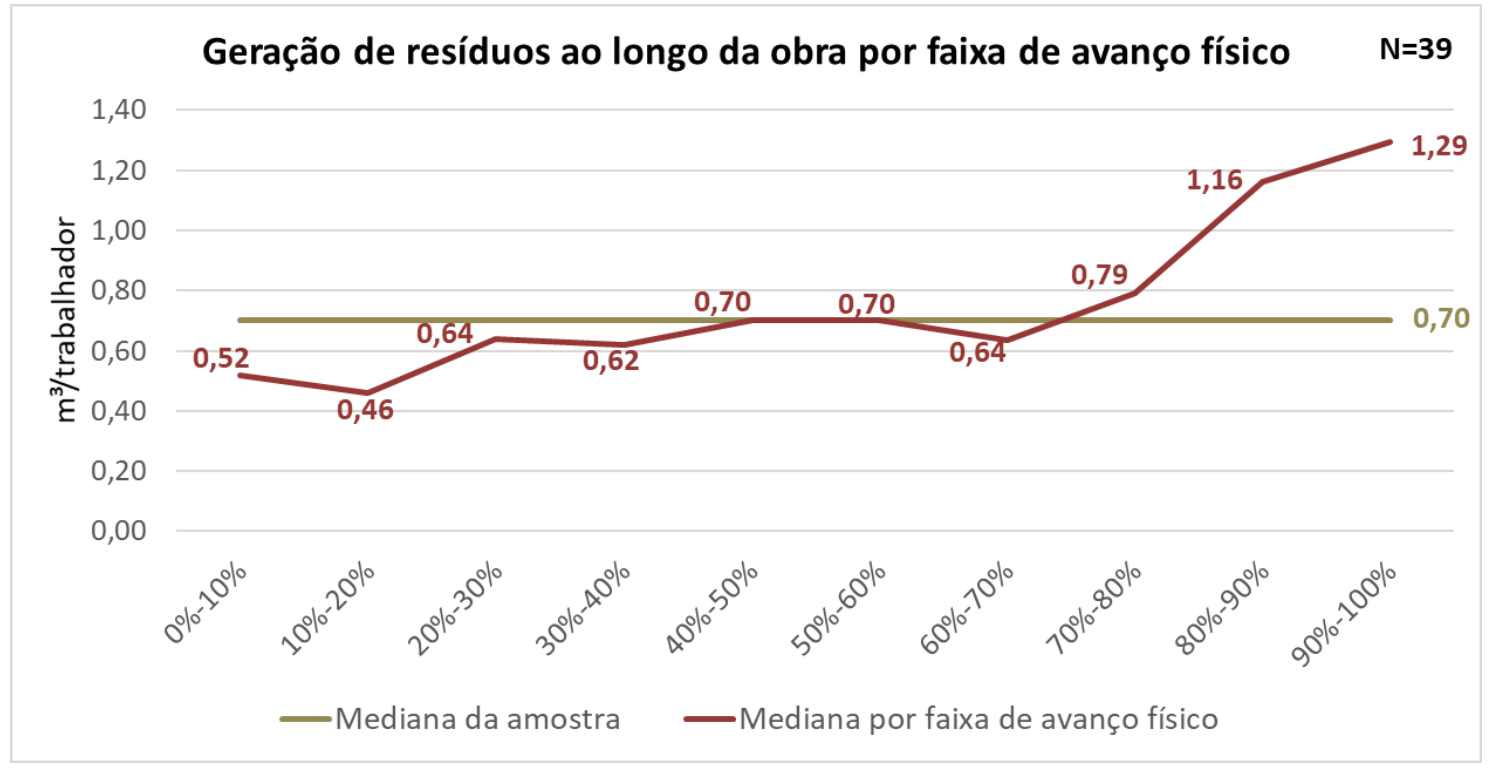

Fonte: Autores

Em relação ao indicador de geração de resíduos ao longo da obra, observou-se uma variação das medianas entre $0,46 \mathrm{~m}^{3} /$ trabalhador e $1,29 \mathrm{~m}^{3} /$ trabalhador. Verifica-se que os menores valores estão associados às fases iniciais da obra (faixas de 0-10\% e 10$20 \%$ ), nas quais grande volume de resíduos são provenientes de solos, que não são considerados no cálculo do indicador, justificando o baixo valor deste nessas faixas. Para as demais faixas de avanço físico, o indicador demonstra uma tendência de crescimento, apresentando seu maior valor para a última faixa (90-100\%), que pode ser devido a fase de desmobilização da obra.

\subsection{Indicadores ao final da obra - Consumo de água, consumo de energia e geração de resíduos}

O Quadro 1 apresenta as análises estatísticas preliminares relacionadas aos indicadores de final de obra, que está associado a metragem construída da obra. Para esses cálculos, foram consideradas obras já finalizadas ou obras nas quais estavam com $85 \%$ ou mais de avanço físico. 
SIBRAGEC - ELAGEC 2019 - de 23 a 25 de Outubro - LONDRINA - PR

Quadro 1 - Valores de referência para os indicadores ao final da obra ( $N=31)$

\begin{tabular}{|c|c|c|c|}
\hline Parâmetro & $\begin{array}{l}\text { Consumo de água } \\
\qquad\left(\mathrm{m}^{3} / \mathrm{m}^{2}\right)\end{array}$ & $\begin{array}{c}\text { Consumo de energia } \\
\left(\mathbf{k W h} / \mathbf{m}^{2}\right)\end{array}$ & $\begin{array}{c}\text { Geração de resíduos } \\
\qquad\left(\mathrm{m}^{3} / \mathrm{m}^{2}\right)\end{array}$ \\
\hline Mínimo & 0,02 & 0,15 & 0,03 \\
\hline Média & 0,24 & 2,64 & 0,14 \\
\hline Mediana & 0,20 & 2,10 & 0,13 \\
\hline Desvio Padrão & 0,17 & 1,95 & 0,07 \\
\hline $\mathrm{CV}$ & $71 \%$ & $74 \%$ & $54 \%$ \\
\hline Q25 & 0,14 & 1,02 & 0,08 \\
\hline Q75 & 0,32 & 3,78 & 0,20 \\
\hline Máximo & 0,65 & 7,13 & 0,30 \\
\hline
\end{tabular}

Fonte: Autores

Para o indicador de consumo de água, o menor valor encontrado foi $0,02 \mathrm{~m}^{3} / \mathrm{m}^{2}$ de área construída, associado a um empreendimento residencial, e o valor máximo obtido foi $0,65 \mathrm{~m}^{3} / \mathrm{m}^{2}$, associado a um empreendimento comercial. Ambos os empreendimentos são de alto padrão e de estrutura de concreto armado moldado no local. A mediana de referência obtida para esse indicador foi $0,20 \mathrm{~m}^{3} / \mathrm{m}^{2}$, apresentando um valor muito próximo da média $\left(0,24 \mathrm{~m}^{3} / \mathrm{m}^{2}\right)$, indicando pouca variabilidade nos dados desse indicador.

Em relação ao indicador de consumo de energia ao final da obra, o menor valor obtido foi $0,15 \mathrm{kWh} / \mathrm{m}^{2}$ e o maior valor obtido foi $7,13 \mathrm{kWh} / \mathrm{m}^{2}$, estando ambos os valores associados a empreendimentos residenciais de alto padrão, com estrutura de concreto armado moldado in loco. A mediana de referência obtida para esse indicador foi 2,10 $\mathrm{kWh} / \mathrm{m}^{2}$ e a média $2,64 \mathrm{kWh} / \mathrm{m}^{2}$.

Para o indicador de geração de resíduos ao final da obra o menor valor encontrado foi $0,03 \mathrm{~m}^{3} / \mathrm{m}^{2}$, valor associado a um empreendimento de estrutura pré-moldada. $\mathrm{O}$ valor máximo encontrado foi $0,30 \mathrm{~m} / \mathrm{m}^{2}$, associado a um empreendimento de alvenaria estrutural. Ambos os empreendimentos são residenciais e fazem parte do programa Minha Casa Minha Vida. A mediana de referência obtida para esse indicador foi 0,13 $\mathrm{m}^{3} / \mathrm{m}^{2}$ e a média $0,14 \mathrm{~m}^{3} / \mathrm{m}^{2}$, sendo estes valores muito próximos, indicando pouca variabilidade nos dados desse indicador.

\section{CONSIDERAÇÕES FINAIS}

Este trabalho apresentou resultados preliminares de um estudo realizado para levantamento de dados e formação de um banco de dados nacional dos indicadores de sustentabilidade do PBQP-H. O levantamento dos dados aconteceu em 6 estados e no Distrito Federal, sendo obtidos, até o momento, dados de 87 obras. As principais contribuições desse estudo são os valores de referência para os seis indicadores, sendo apresentados os indicadores ao longo da obra por faixas de $10 \%$ de avanço físico e os indicadores ao final da obra em valores gerais. O estudo ainda está em desenvolvimento como parte da dissertação de mestrado da autora e de um projeto mais amplo, objetivando uma amostra de 100 obras. Em trabalhos futuros, pretende-se desenvolver análises para cada sistema construtivo obtido na amostra, apresentando valores mais fiéis às particularidades das obras. 
SIBRAGEC - ELAGEC 2019 - de 23 a 25 de Outubro - LONDRINA - PR

\section{REFERÊNCIAS}

BERARDI, U. Sustainability Assessment in the Construction Sector: Rating Systems and Rated Buildings. Sustainable Development. [s. 1.], v. 20, n. 6, p. 411-424, 2012.

COSTA, D. B.; ALVARES, J.S; SILVA, M. B.; SANTOS, V. A. Estudo de valores de referência para os indicadores do PBQP-H voltados à sustentabilidade de canteiros de obra. In: ENCONTRO NACIONAL DE TECNOLOGIA DO AMBIENTE CONSTRU-ÍDO, 17., 2018, Foz do Iguaçu. Anais... Porto Alegre: ANTAC, 2018.

MINISTÉRIO DAS CIDADES. Programa Brasileiro de Qualidade e Produtividade no Habitat. Disponível em: http://pbqp-h.cidades.gov.br/. Acesso em: 14 jul 2018.

SEZER, A.A. "Indicators of environmental and productivity performance for building refurbishment projects". 2013. 29th Annual ARCOM Conference, Reading, 2-4 Sep-tember. Proceedings...Reading, UK, Setembro de 2013.

SILVA, V. G. Avaliação da sustentabilidade de edifícios de escritórios brasilei-ros: diretrizes e base metodológica. 2003. 210 f. Tese (Doutorado em Engenharia Civil) - Escola Politécnica da Universidade de São Paulo, São Paulo.

VAZQUEZ, E. et al. Sustainability in civil construction applied in the construction site phase. WIT Transactions on Ecology and the Environment, [s. 1.], v. 144, p. 265-276, 2011.

YIN, B.C.L.; LAING, R.; LEON, M.; MABON, L. An evaluation of sustainable construction perceptions and practices in Singapore. Sustainable Cities and Society, 39, 613-620. 2018.

YUN, S.; JUNG, W. Benchmarking Sustainability Practices Use throughout Industrial Construction Project Delivery. Sustainability, [s. 1.], v. 9, n. 6, p. 1007, 2017. Disponível em: <http://www.mdpi.com/2071-1050/9/6/1007> 